\title{
Influencia del ph salival en la estabilidad del color de diferentes resinas fluidas: estudio in vitro
}

\author{
Influence of salivary $\mathrm{PH}$ in color stability of different flowable resins: In vitro study \\ Andrea Mejía 1a, Víctor Montaño a, Andrés Viteri ${ }^{2 b}$, Ana Armas ${ }^{10}$.
}

\section{RESUMEN}

Objetivo: Determinar la estabilidad del color de dos diferentes tipos de resina fluida al ser inmersas en saliva artificial con tres $\mathrm{pH}$ diferentes, empleando colorímetro digital Vita Easy-shade. Materiales y métodos: Se plantea un estudio experimental, comparativo e in vitro utilizando 90 discos construidos a partir de dos marcas de resina fluida de $10 \mathrm{~mm}$ de diámetro y $1 \mathrm{~mm}$ de espesor, fotopolimerizados mediante una misma lámpara, tras su confección fueron mantenidos en agua destilada y realizado un primer registro de color utilizando colorímetro digital Easy-shade, las muestras fueron divididas en tres grupos y a su vez en tres subgrupos, pasando a ser sumergidas en saliva artificial con pH diferente de 5,6 y 7 respectivamente durante 7 días, realizando una nueva toma de color mediante colorímetro digital, posteriormente las muestras fueron sumergidas en zumo de remolacha como agente pigmentante, durante 24 horas tras este periodo fueron lavadas para eliminar residuos del pigmento y realizada una tercera toma de color. Resultados: Mayor cambio de color se produjo en los discos que fueron sometidos a saliva artificial con pH $5(\mathrm{p}=0,000)$, los menores cambios de color se presentaron en los discos que fueron sumergidos en saliva artificial con pH 7. Conclusiones: El pH salival 50 ácido, provocó mayor cambio en cuanto al color sin diferencia significativa entre los materiales probados.

Palabras Clave: Acidificación; Color; Resinas compuestas; saliva. (Fuente: DeCS BIREME)

\section{ABSTRACT}

Objective: To determine the color stability of two types of flow composites immersed in artificial saliva with three different $\mathrm{pH}$ values, using Vita Easy-shade digital colorimeter. Materials and methods: An experimental, comparative and in vitro study is proposed using 90 discs made from two fluid resin markings of $10 \mathrm{~mm}$ diameter and $1 \mathrm{~mm}$ thickness, light-cured using the same lamp, after being immersed in distilled water and made a first registration color using Easy-shade digital colorimeter, the samples were divided into three groups and in turn into three subgroups, becoming submerged in artificial saliva with different $\mathrm{pH}$ of 5,6 and 7 respectively for 7 days, making a new color taking by digital colorimeter, later the samples were submerged in beet juice as a pigmenting agent for 24 hours, after this period they were washed to eliminate pigment residues and made a third color take. Results: Greater color change occurred in the discs that were subjected to artificial saliva with $\mathrm{pH} 5(\mathrm{p}=0.000)$, the minor color changes occurred in the discs that were immersed in artificial saliva with $\mathrm{pH} 7$. Conclusions: The $\mathrm{pH}$ salivary 5 or acid, caused greater change in color without significant difference between the materials tested.

Key words: Acidification; Colour; Composite resins; Saliva. (Source: MeSH NLM)

Recibido: 20 de agosto 2018

${ }^{1}$ Universidad Central del Ecuador

2 Universidad Tecnológica Equinoccial

${ }^{a}$ Cirujano dentista

${ }^{\mathrm{b}}$ Magister

${ }^{\mathrm{c}} \mathrm{PhD}$, docente

Correspondencia:

Andrea Mejía

Correo electrónico: andre_nat13@hotmail.com
Aprobado: 18 de diciembre de 2018

Publicado: 06 de julio de 2019

Este es un artículo Open Access distribuido bajo la licencia Creative Commons Atribución-NoComercialCompartirlgual 4.0 


\section{INTRODUCCIÓN}

En la práctica clínica diaria las resinas compuestas constituyen los materiales restauradores directos más frecuentemente empleados, sin embargo, su desempeño se encuentra en dependencia de su técnica de empleo ${ }^{(1)}$ composición (2) y ciertos hábitos de dieta e higiene relacionados estrechamente con el paciente ${ }^{(3)}(4)$, pudiendo desencadenar alteraciones en la rugosidad superficial al crear espacios para la acumulación de placa y absorción de pigmentos ${ }^{(5)}$ repercutiendo directamente en la estabilidad del color del material restaurador ${ }^{(6)}$.

La saliva se presenta como un fluido importante para las funciones de masticación, fonación, deglución (7), interviniendo en procesos de inmunidad, reacciones enzimáticas, soporte celular, tensión y flexibilidad tisular (8) puede afectarse en cuanto su pH por causas fisiológicas o adquiridas ${ }^{(9)}$;provocando daños severos en los tejidos bucales duros y blandos, así como en las restauraciones afectando principalmente la estabilidad cromática del material actuando a nivel de las aminas (10) (11) encargadas de activar la polimerización.

La dieta principalmente ácida en la población adulta joven y la ausencia de datos que evalúen la influencia del $\mathrm{pH}$ salival sobre el desempeño de las resinas compuestas, hacen que se pretenda mediante este estudio evaluar el cambio de color que se presenta en resinas compuestas fluidas cuando son sometidas a diferentes $\mathrm{pH}$ salivales ${ }^{(12)}$.

\section{MATERIALES Y METODOS}

Noventa discos construidos a partir de una matriz de poli acrílico de $10 \mathrm{~mm}$ de diámetro $1 \mathrm{~mm}$ de espesor, con dos tipos diferentes de resina fluida (n:45) AlpHaFlow (AlpHa- Dent, Filipinas) y Opallis Flow (FGM, Brasil) dosificado directamente de la jeringa hasta llenar las matrices, foto polimerizados mediante el uso de una lámpara de luz led (Eliper S10, 3M-ESPE), durante 40 segundos directamente sobre la superficie de la muestra; pulidos mediante discos de lija-Sof-lex (3M Oral, EEUU), en secuencia decreciente de granulación durante 15 segundos, utilizando un contraángulo de baja velocidad con movimientos intermitentes.

Estos discos fueron examinados cuidadosamente mediante microscopio estereoscópico verificando ausencia de burbujas, irregularidades o grietas. Los discos considerados idóneos permanecieron almacenados en recipientes oscuros y sumergidos en agua destilada durante 24 horas, tras lo cual fue realizada la medición de color por medio de un colorímetro digital Vita Easy shade (VITA, Alemania)
En base a la guía de colores Vita Classical (VITA), ordenada de acuerdo al matiz A1-D4.

Los discos debidamente identificados fueron subdivididos en 3 subgrupos (n:15) depositando según el grupo, el espécimen en $18 \mathrm{ml}$ de saliva artificial modificada químicamente utilizando bicarbonato de sodio y ácido cítrico, hasta obtener saliva con un $\mathrm{pH} 5$, 6 y 7 empleando un pH-metro digital (ATC, Etekcity) como elemento evaluador de $\mathrm{pH}$. Se realizó cambios de la saliva cada 24 horas, durante siete días, luego de lo cual fue realizada una segunda evaluación de color.

Los especímenes fueron colocados en $18 \mathrm{ml}$ de zumo de remolacha, obtenido previamente, por trituración y colado de 100 gramos del tubérculo crudo, permaneciendo en esta sustancia durante 24 horas, tras lo cual una nueva evaluación de color de cada disco fue realizada.

Los valores obtenidos en las tres mediciones de color fueron recolectados y calculada la diferencia entre los tiempos considerándose el inicial y la segunda medición como un tiempo y los valores obtenidos entre inicial y final como un segundo tiempo, esta diferencia sometida a análisis estadístico mediante el programa estadístico SPSS.

\section{RESULTADOS}

La prueba de normalidad Kolmogorov-Smirnov realizada con los datos, comprobaron un nivel de significancia, menor que 0,05 , que reveló no provenir de una población con distribución normal, razón por la cual fueron ejecutadas pruebas no paramétricas de Kruskal Wallis para conocer la existencia de diferencias de color debido al $\mathrm{pH}$ salival dentro de los grupos y Mann Whitney para establecer comparación entre los dos grupos.

Al evaluar las medias obtenidas y comparar las mediciones de color realizadas, se encontraron diferencias en cuanto a cambios de color entre las resinas y entre los $\mathrm{pH}$ en los períodos evaluados, destacándose siempre cambios más evidentes en las muestras sometidas al contacto con saliva de $\mathrm{pH} 5$ (Tabla 1).

Buscando determinar diferencias entre los grupos se realizó la prueba de la $U$ de Mann Whitney, observándose diferencia de color significativa, entre los grupos, siendo más evidente el cambio de color en las muestras que permanecieron sometidas a saliva artificial con $\mathrm{pH} 5$ tanto en los dos periodos $(\mathrm{p}=0,000)$ que se corrobora con la prueba de Kruskal Wallis, sin diferencia entre las dos resinas evaluadas 
Tabla 1. Comparación de medidas entre las resinas y entre los $\mathrm{pH}$ en los períodos evaluados

pH

Medición inicial

Medición inicial
Resina Opalis

Media $p$

Media $p$

Resina Alpha

Media $p$ Resina Opalis

$\mathrm{pH} 5$

\begin{tabular}{llll}
\hline 7,67 & 0,667 & 6,07 & 0,621 \\
\hline 4,53 & 0,496 & 4,07 & 0,441
\end{tabular}

$\mathrm{pH} 6$

$4,07 \quad 0,441$

$\mathrm{pH} 7$

2,73

0,228

2,53

0,215

$4,22 \quad 0,338$

Media $\quad p$

$\begin{array}{llll}13,67 & 0,386 & 10,60 & 0,496 \\ 10,3 & 0,504 & 8,33 & 0,287\end{array}$

$\begin{array}{llll}5,33 & 0,735 & 4,87 & 0,710\end{array}$

$9,78 \quad 0,605 \quad 7,93 \quad 0,463$

\section{DISCUSIÓN}

El pH salival 5 considerado ácido produjo una mayor alteración en la estabilidad cromática de las resinas fluidas, resultados que guardan relación con estudios previos donde compararon la estabilidad en cuanto al color de tres resinas fluidas de diferentes marcas sometidas a tres tipos de $\mathrm{pH}$ salival $(6.5,7$ y 7.5$)$ destacando cambio máximo de color cuanto más ácido fuera el $\mathrm{pH}{ }^{(13)}$, resultado que concuerda con el obtenido en el presente estudio, donde frente al pH 5 salival que fue el más ácido, se presentaron cambios notables sobre las muestras mientras que, cuando las muestras fueron sumergidas en saliva con $\mathrm{pH} 7$ se evidenció cambios de color en menor proporción independientemente de la resina empleada en el estudio.

Si bien está demostrado que el $\mathrm{pH}$ ácido de algunas sustancias pueden degradar la superficie de las resinas creando micro porosidades para el depósito de pigmentos y por ende el cambio de color será más evidente ${ }^{(14)}$, este estudio pretendió evaluar estos cambios, cuando las muestras que ya permanecieron 7 días sumergidas en saliva con $\mathrm{pH} 5$ y $\mathrm{pH} 6$ fueron inmersas 24horas en zumo de remolacha; considerando que la remolacha podía representar diferentes sustancias de consumo habitual por una persona, encontrándose en sus resultados que existieron cambios significativos de color asociados al contacto con la saliva con pH ácido. Siendo la avidad bucal un hábitat muy desfavorable para los materiales restauradores éstos se muestran susceptibles de alterarse, agentes exógenos cómo alimentos, bebidas gaseosas, hábitos dañinos como el cigarrillo y el alcohol, provocan en las resinas manchas exógenas ${ }^{(15)}$; la falta de conocimiento sobre los posibles efectos de la saliva y su $\mathrm{pH}$ dificulta el desempeño del profesional odontólogo y por ende el adecuado criterio en la selección adecuada del material a utilizar; por otro lado, el paciente al desconocer esto descuida su alimentación, desencadenando a largo plazo cambios desfavorables $y$ disminución en la durabilidad y calidad del trabajo del odontólogo ${ }^{(16)}$.

Evaluaciones del efecto del refresco de maíz morado en el color de una resina compuesta, establecen que el efecto del mencionado sobre el cambio de color de las resinas compuestas se debe a que posee un pigmento vacuolar denominado antocianina por lo que el cambio de color es mayor (17), esto concuerda con los resultados obtenidos en el presente estudio ya que después de permanecer 24 horas las muestras sumergidas en zumo de remolacha el cambio de color en las mismas fue aún más evidente asociando estos cambios al pigmento que posee la remolacha denominado betalaína que es la responsable del color rojo del fruto ${ }^{(18)}$, si bien; en este estudio se empleó como medio de pigmentación considerando su uso más frecuente, sin embargo, queda la posibilidad del 
empleo de otros colorantes naturales o artificiales para futuras investigaciones.

Si bien es cierto, las propiedades tanto físicas como mecánicas de las resinas depende de los componentes de las mismas; mientras menor sea el contenido de relleno inorgánico la resina se presentará menos estable en su color, esto es porque al poseer mayor porcentaje de matriz orgánica existirá mayor absorción de agua (19) lo que provoca una degradación hidrolítica, lo que desencadena que exista una unión débil entre la matriz orgánica y el relleno inorgánico de la resina alterando el color de las mismas (20), los resultados obtenidos en esta investigación evidencian que las muestras constituidas por una resina con mayor contenido de matriz orgánica y menor relleno inorgánico fueron menos estables en su color independientemente del $\mathrm{pH}$ y sustancia a la que fueron sometidas a pesar de que todas las muestras fueron conformadas por resina fluida, esto se cree que fue debido a que la resina con menor contenido de relleno absorbió mayor cantidad de agua al poseer mayor cantidad de matriz orgánica en su composición.

Durante el proceso de pulido las partículas de relleno se pueden desprender dejando porosidades que serán mayores o menores en tamaño dependiendo del tamaño de las partículas de relleno desprendidas por lo que aumentará acumulación de pigmentos (21); la literatura establece que el acabado y pulido de las resinas es un paso indispensable para evitar irregularidades en la superficie de las resinas (22), en este estudio se realizó el pulido de todas las muestras verificando que todas cuenten con una superficie lisa para uniformizar las condiciones por lo que se cree que el cambio de color en las muestras de este estudio no se atribuye a la técnica de pulido sino, al medio ácido al que fueron sometidas las muestras así como también, a la composición química de las resinas que se utilizaron.

Como clínicos es básico considerar el posible efecto que el pH salival puede tener en el cambio de color de las resinas, la dieta acida principalmente se ha convertido en la favorita por la población adulta joven lo cual puede desencadenar problemas estéticos y funcionales de importancia, razón por la cual es básico el asesoramiento en hábitos, guiando una dieta equilibrada para mantener un ph salival neutro y un flujo salival regular $y$ de calidad, lamentablemente la gran mayoría de resinas que el mercado ofrece cuentan con metacrilato en su composición por lo que la susceptibilidad a presentar estos fenómenos de degradación es alta.

En las condiciones que este estudio in vitro fue ejecutado, se puede concluir que el pH salival 50 ácido, provocó mayor cambio en cuanto al color de las dos resinas compuestas evaluadas. Por lo que es necesario educar al paciente sobre la dieta a consumir, evitando que el pH salival se torne ácido $y$ afecte la integridad y estética de las restauraciones dentales.

Contribuciones de autoría: NVCT, TACR y RMV diseñaron el estudio, recopilaron y analizaron los datos. Los autores redactaron y aprobaron el manuscrito.

Fuente de financiamiento: Autofinanciado.

Conflicto de intereses: Los autores declararon no tener conflictos de interés.

\section{REFERENCIAS}

1. Randolph LD, Palin WM, Leloup G, Leprince JG. Filler characteristics of modern dental resin composites and their influence on physico-mechanical properties. Dent Mater. 2016 Dec; 32(12):1586-1599.

2 Kocaağaoğlu $H$, Aslan $T$, Gürbulak A, Albayrak $H$, Taşdemir Z, Gumus H. Efficacy of Polishing Kits on the Surface Roughness and Color Stability of Different Composite Resins. Nig J of Clinic Practice. 2017 May; 20(5):557-565.

3. Habib AN, Abdelmoniem SA, Mahmoud SA. Effect of Children's Drinks on Color Stability of Different Dental Composites: An in vitro Study. J Clin Pediatr Dent. 2017;41(2):120-125.

4. Dias HB, Carrera ET, de Souza AN. The influence of $\mathrm{pH}$ and chemical composition of beverages on color stability of a nanofilled composite resin. Gen Dent. 2016 Dec;64(6):21-27.

5. Alfawaz Y. Impact of Polishing Systems on the Surface Roughness and Microhardness of Nanocomposites. J Contemp Dent Pract. 2017 Aug 1;18(8):647-651.

6. Karadas M. The effect of different beverages on the color and translucency of flowable composites. Scanning. 2016 Nov;38(6):701-709.

7. Dawes C, Pedersen AM, Villa A, Ekström J, Proctor GB, Vissink $A$, et al. The functions of human saliva: $A$ review sponsored by the World Workshop on Oral Medicine VI. Arch Oral Biol. 2015 Jun;60(6):863-74.

8. Neyraud E. Role of saliva in oral food perception. Monogr Oral Sci. 2014; 24:61-70.

9. Yakubov GE. Lubrication. Monogr Oral Sci. 2014; 24:7187.

10. Delaviz Y, Finer Y, Santerre JP. Biodegradation of resin composites and adhesives by oral bacteria and saliva: a rationale for new material designs that consider the clinical environment and treatment challenges. Dent Mater. 2014 Jan;30(1):16-32. 
11. Camargo FM, Della Bona Á, Moraes RR, Coutinho de Souza CR, Schneider LF. Influence of viscosity and amine content on $\mathrm{C}==\mathrm{C}$ conversion and color stability of experimental composites. Dent Mater. 2015 May; 31(5):10915.

12. Khan AA, Siddiqui AZ, Al-Kheraif A, Zahid A, Divakar DD. Effect of different $\mathrm{pH}$ solvents on micro-hardness and surface topography of dental nano-composite: An in vitro analysis. Pak J Med Sci. 2015 Aug; 31(4):854-859.

13. Batra $R$, Kataria $P$, Kapoor S.Effect of Salivary $\mathrm{pH}$ on Color Stability of Different Flowable Composites - A Prospective In-vitro Study. J Clin Diagn Res. 2016 Oct;10(10):43-46.

14. Kitasako Y, Sasaki Y, Takagaki T, Sadr A, Tagami J. Age-specific prevalence of erosive tooth wear by acidic diet and gastroesopHageal reflux in Japan. J Dent. 2015 Apr;43(4):418-23.

15. Saijai T, Kukiattrakoon B, Siriporananon C, Ornprasert N, Mettasitthikorn W, Likhitpreeda S, et al. The effect of different beverages on surface hardness of nanohybrid resin composite and giomer. J Conserv Dent. 2014; 17(3):261-265.

16. Ardu S, Duc O, Di Bella E, Krejci I, Daher R.Color stability of different composite resins after polishing. Odontology. 2018;106(3):328-333.
17. Acuña E, Delgado C, Mg Tay. Efecto del refresco de maíz morado en el color de una resina compuesta. RODYB [Internet]. 2016: 5(2): 3439.

18. Kagami $\mathrm{H}$, Kurata $\mathrm{M}$, Matsuhira $\mathrm{H}$, Taguchi $\mathrm{K}$, Mikami T, Tamagake H, et al. Sugar beet (Beta vulgaris L.). Methods Mol Biol. 2015; 1223:33547.

19. Kaleem M, Watts DC. Effect of filler particles morphology of resin-composites on cavity packing force for repeated condensation. Dent Mater J. 2017 May 31;36(3):340-347.

20. Zhu Z, Zhu YQ. Comparative evaluation of marginal microleakage of three different resins in Class V composite restorations. Shanghai Kou Qiang Yi Xue. 2017 Jun;26(3):241-245.

21. Kumari RV, Nagaraj H, Siddaraju K, Krishna P. Evaluation of the Effect of Surface Polishing, Oral Beverages and Food Colorants on Color Stability and Surface Roughness of Nanocomposite Resins. J of International Oral Health. 2015 Jul; 7(7):63-70.

22. Alfawaz Y. Impact of Polishing Systems on the Surface Roughness and Microhardness of Nanocomposites. J Contemp Dent Pract. 2017 Aug 1;18(8):647-651. 